\title{
Time Trends in Incidence of Pathological and Etiological Stroke Subtypes during 16 Years: The Erlangen Stroke Project
}

\author{
Peter L. Kolominsky-Rabas ${ }^{a}$ Silke Wiedmann ${ }^{b}$ Michael Weingärtner ${ }^{a}$ \\ Thomas G. Liman ${ }^{c}$ Matthias Endres ${ }^{c}$ Stefan Schwab ${ }^{d}$ Michael Buchfelder ${ }^{\mathrm{e}}$ \\ Peter U. Heuschmann ${ }^{b, f}$

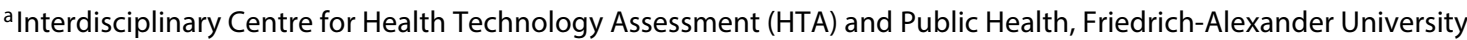 \\ of Erlangen-Nürnberg, Erlangen-Nürnberg, ${ }^{\mathrm{b}}$ Institute of Clinical Epidemiology and Biometry, Comprehensive Heart \\ Failure Center, University of Würzburg, Würzburg, ' Department of Neurology, Center for Stroke Research, German \\ Center for Neurodegenerative Diseases (DZNE), German Center for Cardiovascular Research (DZHK), Partner Site, \\ Charité - Universitätsmedizin Berlin, Berlin, ${ }^{\mathrm{d} D e p a r t m e n t}$ of Neurology, ${ }^{\mathrm{e} D e p a r t m e n t}$ of Neurosurgery, University \\ Hospital Erlangen, Erlangen, and ${ }^{\mathrm{f} C l i n i c a l}$ Trial Center Würzburg, University Hospital Würzburg, Würzburg, Germany
}

\section{Key Words}

Stroke $\cdot$ Epidemiology $\cdot$ Incidence $\cdot$ Time trends $\cdot$ Register

\begin{abstract}
Background: Population-based data, which continuously monitors time trends in stroke epidemiology are limited. We investigated the incidence of pathological and etiological stroke subtypes over a 16 year time period. Methods: Data were collected within the Erlangen Stroke Project (ESPro), a prospective, population-based stroke register in Germany covering a total study population of 105,164 inhabitants (2010). Etiology of ischemic stroke was classified according to the Trial of Org 10172 in Acute Stroke Treatment (TOAST) criteria. Results: Between January 1995 and December 2010, 3,243 patients with first-ever stroke were documented. The median age was 75 and $55 \%$ were females. The total stroke incidence decreased over the 16 year study period in men (Incidence Rate Ratio 1995-1996 vs. 2009-2010 (IRR) 0.78; $95 \% \mathrm{Cl} 0.58-0.90)$ but not in women. Among stroke sub-
\end{abstract}

types, a decrease in ischemic stroke incidence (IRR 0.73; 95\% $\mathrm{Cl}$ 0.57-0.93) and of large artery atherosclerotic stroke (IRR $0.27 ; 95 \% \mathrm{Cl} 0.12-0.59$ ) was found in men and an increase of stroke due to small artery occlusion in women (IRR 2.33; 95\% $\mathrm{Cl}$ 1.39-3.90). Conclusions: Variations in time trends of pathological and etiological stroke subtypes were found between men and women that might be linked to gender differences in the development of major vascular risk factors in the study population.

(c) 2015 S. Karger AG, Basel

\section{Background}

Due to the ageing population, the absolute number of stroke patients is expected to increase substantially in the next decades if age-specific incidence rates remain stable $[1,2]$. Simulation studies showed that the absolute number of stroke patients can be reduced if effective preventive measures in the population were applied for primary

\section{KARGER 125}

(c) 2015 S. Karger AG, Base

$0251-5350 / 15 / 0441-0024 \$ 39.50 / 0$
Peter L. Kolominsky-Rabas, MD, PhD, MBA, Erlangen Stroke Project (ESPro) National Information System of the Federal Health Monitoring, Interdisciplinary Centre for Health Technology Assessment (HTA) and Public Health

Friedrich-Alexander University of Erlangen-Nürnberg Schwabachanlage 6

DE-91052 Erlangen (Germany), E-Mail peter.kolominsky@uk-erlangen.de 
or secondary stroke prevention [3]. Therefore, data monitoring time trends in the incidence and underlying pathomechanisms of stroke are useful to inform health policy decision makers about the effectiveness of prevention strategies in the population. Previous studies on time trends in stroke incidence during the last years reported conflicting results [4-9]. Population-based data continuously monitoring incidence of etiological stroke subtypes within the same source population over a time span of more than one decade are scarce. In addition, time trends related to the incidence of ischemic stroke that can be obtained using an established mechanism-based classification scheme such as the Trial of Org 10172 in Acute Stroke Treatment criteria (TOAST) [10] are lacking.

We investigated 16-year time trends in the incidence of pathological and etiological stroke subtypes using a standardized pathological and etiological mechanismbased classification system within a population-based stroke register. The incidence of stroke subtypes within the same study population in Germany was continuously monitored.

\section{Material and Methods}

The Erlangen Stroke Project (ESPro) is an ongoing prospective community-based stroke register in Germany, which has continuously monitored stroke incidence in a study population of 105,164 inhabitants (census 2010) since 1994. The characteristics of the study population, methods of assessment, and investigations have been described in detail elsewhere [11].

\section{Data Collection}

From January 1995 to December 2010, all hospitalized and nonhospitalized patients in the study area with suspected fatal or nonfatal stroke or transient ischemic attack were identified. To ensure completeness of case ascertainment, standardized criteria were applied with details published previously [11, 12]. To identify patients admitted to hospitals, daily checks of hospital admission and discharge records were made in the study area. In addition, nursing and residential homes were checked for identifying nonhospitalized stroke patients. For an accurate estimate of the number of nonfatal strokes that were managed outside the hospital, the general practitioners in the community were contacted regularly. There were no major changes in case ascertainment methods during the study period. Special trained study nurses and research assistants collected all data prospectively following standardized operating procedures that were stable over the whole documentation period.

\section{Data Definition}

Stroke diagnosis was defined by a study clinician according to the criteria of the World Health Organization [13]. Patients with first-ever-lifetime-stroke were included in the present study [14]. Classification of pathological stroke subtype (ischemic stroke
[IS], primary intracerebral hemorrhage [PICH], and subarachnoid hemorrhage [SAH]) was established by means of brain imaging (CT or MRI scan) or necropsy examination. All patients with stroke in whom imaging or post-mortem examination could not be performed or in whom the results of the brain imaging were unknown were classified as unspecified stroke [UNS]. For determination of etiological subtype of ischemic stroke, the original TOAST (Trial of Org 10172 in Acute Stroke Treatment) criteria were used [10] with details of evaluation of TOAST classification in the ESPro described previously [12]. Categories of the TOAST classification are large-artery atherosclerosis (LAA), including large-artery thrombosis and artery-to-artery embolism; cardioembolism (CE); small artery occlusion (SAO); stroke of other determined cause (OC); and stroke of undetermined cause (UND). The ratings were performed by trained staff considering the results of all investigations and the neurological examination.

\section{Statistical Analysis}

The source population of the ESPro was estimated based on data from the constantly updated official population register of Erlangen. Crude incidence rates were calculated for age group, sex, pathological and etiological stroke subtypes; sex and stroke subtype incidence rates were age-adjusted to the standard European population [15]. Data were reported in two-year time intervals. To enable comparisons, time trends during the 16 year observation period were compared with the first period (1995-1996) by calculating Incidence Rate Ratios (IRR); 95\% CI for the direct standardized IRR, calculated by the delta method [16]. Five patients with unknown sex were excluded. The TOAST classification was performed by specifically trained raters considering the results of all diagnostic investigations and the neurological examination. For testing inter-observer reliability of the TOAST classification, twenty randomly selected patients were classified by two independent raters between 1995 and 1998 [12] and additionally forty patients after 1999. Inter-observer reliability of classification among raters was good for both time intervals (unweighted $\kappa 0.65,95 \%$ CI $0.35-0.96$ and $0.63,95 \%$ CI $0.43-0.83$, respectively). Statistical analyses were performed with SAS software version 9.2 (SAS Institute Inc., Cary, N.C., USA).

\section{Ethics}

The design of the study was approved by the local ethics committee. Patients or their legal representatives gave their written informed consent to participate.

\section{Results}

Between January 1995 and December 2010, 3,243 patients with first-ever stroke were registered. The median age was 75 years (Interquartile range 66-82), 1,787 (55\%) were females. Changes of the source population regarding socio-demographic characteristics, and stroke subtype over the 16-year study period by sex are shown in online supplementary table A (for all online suppl. material, see www.karger.com/doi/10.1159/000371353). 


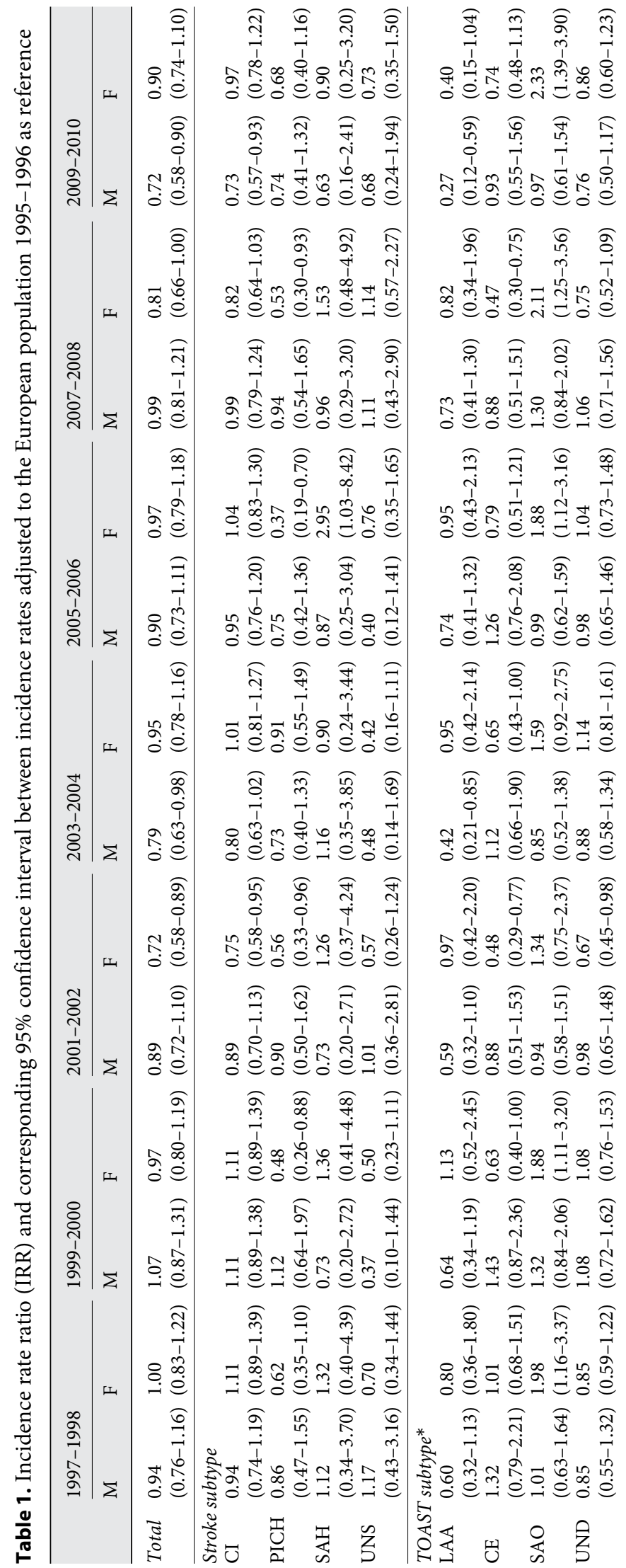

\section{Frequency of Stroke Subtypes}

The distribution of pathological subtypes was as follows: ischemic stroke (IS) 2,582 (79.7\%); primary intracerebral hemorrhage (PICH) 394 (12.2\%); subarachnoid hemorrhage (SAH) 96 (3.0\%), and 166 (5.1\%) were of unspecified stroke (UNS).

Overall, it was possible to classify ischemic stroke etiology according to the TOAST classification in 2,486 out of 2,582 patients with IS (96.3\%). The distribution of etiological ischemic stroke subtypes of patients being classified was as follows: LAA 246 (9.9\%); CE 609 (24.5\%); SAO 654 (26.3\%); OC 43 (1.7\%); UND 934 (37.6\%) of patients with $\mathrm{OC}$ ischemic stroke was caused by the following reasons: fibro-muscular dysplasia 9 (20.9\%); dissection 11 (25.6\%); thrombotic disorders 8 (18.6\%); migraine 2 (4.7\%); vasculitis 5 (11.6\%); other causes 8 (18.6\%).

\section{Stroke Incidence}

Biannual stroke incidence rates per 100,000 age-adjusted to the European population are presented in online supplementary table B. Time trends in age-standardized incidence rates are shown in table 1 . Total stroke incidence decreased over the 16 year study period in men (incidence rate ratio 1995-1996 vs. 2009-2010 (IRR) $0.72 ; 95 \%$ CI $0.58-0.90$ ) but not in women (IRR 0.90; $95 \%$ CI 0.74-1.10). Among pathological stroke subtypes, a decrease in ischemic stroke incidence was found in men (IRR 0.73; 95\% CI 0.57-0.93). Incidence of LAA stroke decreased in men (IRR 0.27; 95\% CI 0.12-0.59) and incidence of SAO increased in women (IRR 2.33; 95\% CI 1.39-3.90).

\section{Discussion}

In our study a moderate reduction in overall stroke incidence was found for men but not in women over the 16 year study period. Incidence of ischemic stroke decreased in men, with no other major changes in incidence of different stroke subtypes observed. Regarding ischemic stroke etiology, a reduction of incidence of LAA was seen in men and an increase of SAO in women.

A systematic review on world-wide stroke incidence trends found a decrease in overall age-adjusted stroke incidence rates over the last forty years in the developed countries [17]. For the last decade, however, data on temporal trends of age- and sex-adjusted stroke incidence in European populations is limited [1]. Moreover, the estimation of time-trends across different population-based registers might be difficult as these studies are heteroge- 
neous in a number of factors affecting stroke incidence in the local population such as geographical region, ethnic group diversity or risk factor distribution in the general population. Population-based studies reporting time trends in stroke incidence in the same source population since the mid-1990s demonstrated decreasing rates in the United Kingdom [4-6], the United States [7], New Zealand [8] in mainly white as well as in multi-ethnic populations. No changes in stroke rates however were observed in black US Americans [7], while an increase in incidence was found in 'pacific people' in New Zealand [8], France [9] and some parts of Eastern Europe [18, 19]. There are possible explanations for these differences in time trends of age-adjusted incidence rates of populationbased studies during the last years. A higher age-adjusted annual stroke incidence rate was found in stroke registers that reported a decrease of stroke incidence over time [5, $6,8]$. Compared to our data, that might indicate that our population already reached a 'floor' effect in stroke incidence. Major ethnic differences of source populations may also contribute to this heterogeneity in variations of stroke risk over time as registers including multi-ethnic populations such as the SLSR or the ARCOS reported significant ethnic group disparities in incidence trends $[5,8]$.

Data from previous population-based studies on time trends regarding pathological subtypes suggest a decrease in primary intracerebral hemorrhage (PICH) during the last 20 years in high-income countries [17]. However, in line with our findings, no major time trends in $\mathrm{PICH}$ incidence was found in the white SLSR population and in the Dijon Register between 1985 and 2004 [4, 20]. The lacking decrease in incidence of PICH in the ESPro from 1995 to 2010 might be caused by stable prevalence rates of hypertension in the population, one of the major risk factors for PICH. In a recent review of representative cross-sectional surveys on vascular risk factors in North America and Europe, Germany is considered to be one of the regions with the highest prevalence of hypertension [21] that was also confirmed by recent data from the general population $[21,22]$. In line with our findings, time trends of subarachnoid hemorrhage (SAH) are showing stable incidence rates during the last decades [17].

Regarding the etiology of ischemic stroke subtypes, we observed in our study population a reduction of LAA incidence in men and an increase of SAO in women. To the best of our knowledge, data on time trends in incidence of etiological stroke subtypes from population-based studies using an established mechanism-based classification system such as the TOAST classification are lacking so far. Improved primary preventive treatment and a

16-Year Time Trends in Stroke Subtype Incidence healthier lifestyle during the last two decades in our population might account for the finding in men. For example, an increase in the uptake of statin therapy was observed in the recent years as well as a decrease of smoking in the general population $[23,24]$. The higher incidence of SAO in women might be caused by an increase in the prevalence of risk factors for this subtype such as hypertension, which is highly prevalent in the general older aged population with rather stable prevalence rates in Germany [22]. The life expectancy of people from the source population increased during the study period. However, as we are reporting age-standardized rates, the effect of increasing age in our source population should be controlled for. Similar to other population-based stroke registers [20], we could not observe an increase in the rate of cardioembolic strokes over time. As cardioembolic stroke due to atrial fibrillation occurs often in older age groups, especially in females, this might be explained by the fact that there were no major changes in the median age at stroke onset in the register.

Our study has limitations. One limitation represents the small sample size of our source population, which resulted in wide confidence intervals of the respective estimates. In addition, due to the design of the study we were not able to link the incidence data with data on treatment and control of major vascular risk factors from the general local population. No data were available for assessing potential time trends in the use of statins, antithrombotic agents or carotid surgery in the general population. We cannot exclude that changes in the used diagnostic techniques over the 16 year time period might have contributed to some of our findings; for example, due to the wider availability and the better diagnostic accuracy of diagnostic techniques such as extra-cranial imaging. Finally, some limitations of the mechanism-based TOAST classification system need to be addressed, such as the high rates of undefined stroke due to missing distinctions between undefined and concurrent etiology as well as the noninclusion of new pathophysiological and diagnostic knowledge. Novel adaptations of the TOAST criteria have been proposed, which were not available at the time the study was initiated $[25,26]$.

\section{Conclusion}

Variations in time trends of pathological and etiological stroke subtypes were found between men and women that might be linked to gender differences in the development of major vascular risk factors in our population. 


\section{Acknowledgments}

The authors thank their fellow participants of the Erlangen Stroke Project: Universitätsklinikum Erlangen, Waldkrankenhaus St. Marien, Klinikum am Europakanal, the General Practitioners Association Erlangen and the Regional Public Health Office of Erlangen. We would like to thank the 100 general practitioners, their staff, and the patients and their families. The authors thank Ch. Kriza for the language review as 'native speaker'.

\section{Funding}

The data collection is supported (IIA5-2013-2513KEU305) by the German Federal Ministry of Health (BMG) as part of the National Information System of the Federal Health Monitoring (Gesundheitsberichterstattung des Bundes - GBE).

\section{Disclosure Statements}

P.L.K.-R. received funding from: German Federal Ministry of Research and Education (BMBF), European Commission, Bavarian State Research Foundation, and University of Erlangen-Nürnberg.

P.U.H. received research support from: German Federal Ministry of Research and Education (BMBF), European Union, German Stroke Foundation, Charité - Universitätsmedizin Berlin, Berlin Chamber of Physicians, and University Hospital of Würzburg.

S.W. from: BMBF.

T.G.L. from the DFG (excellence cluster NeruoCure) and the Stiftung Charité (Clincial Scientist program).

M.E. receives funding from the DFG (Excellence cluster NeuroCure; SFB TR 43, KFO 247, KFO 213), BMBF (Centre for Stroke Research Berlin), EU (European Stroke Network, WakeUp, Counterstroke), Volkswagen Foundation (Lichtenberg Program), Corona Foundation.

\section{References}

$\checkmark 1$ Feigin VL, Forouzanfar MH, Krishnamurthi R, Mensah GA, Connor M, Bennett DA, Moran AE, Sacco RL, Anderson L, Truelsen T, O'Donnell M, Venketasubramanian N, Barker-Collo S, Lawes CM, Wang W, Shinohara Y, Witt E, Ezzati M, Naghavi M, Murray C; Global Burden of Diseases, Injuries, and Risk Factors Study 2010 (GBD 2010) and the GBD Stroke Experts Group: Global and regional burden of stroke during 1990-2010: findings from the global burden of disease study 2010. Lancet 2014;383:245-254

- 2 Kolominsky-Rabas PL, Heuschmann PU, Marschall D, Emmert M, Baltzer N, Neundorfer B, Schoffski O, Krobot KJ: Lifetime cost of ischemic stroke in Germany: results and national projections from a population-based stroke registry: the Erlangen Stroke Project. Stroke 2006;37:1179-1183.

3 Hallstrom B, Jonsson AC, Nerbrand C, Norrving $B$, Lindgren $A$ : Stroke incidence and survival in the beginning of the 21 st century in southern Sweden: comparisons with the late 20th century and projections into the future. Stroke 2008;39:10-15.

4 Heuschmann PU, Grieve AP, Toschke AM, Rudd AG, Wolfe CD: Ethnic group disparities in 10-year trends in stroke incidence and vascular risk factors: the South London Stroke Register (SLSR). Stroke 2008;39:22042210.

5 Wang Y, Rudd AG, Wolfe CD: Age and ethnic disparities in incidence of stroke over time: the South London Stroke Register. Stroke 2013;44:3298-3304.

-6 Rothwell PM, Coull AJ, Giles MF, Howard SC, Silver LE, Bull LM, Gutnikov SA, Edwards P, Mant D, Sackley CM, Farmer A, Sandercock PA, Dennis MS, Warlow CP, Bamford JM, Anslow P: Change in stroke incidence, mortality, case-fatality, severity, and risk factors in Oxfordshire, UK from 1981 to 2004 (Oxford Vascular Study). Lancet 2004;363: 1925-1933.

7 Kleindorfer DO, Khoury J, Moomaw CJ, Alwell K, Woo D, Flaherty ML, Khatri P, Adeoye O, Ferioli S, Broderick JP, Kissela BM: Stroke incidence is decreasing in whites but not in blacks: a population-based estimate of temporal trends in stroke incidence from the greater Cincinnati/Northern Kentucky Stroke Study. Stroke 2010;41:13261331.

-8 Carter K, Anderson C, Hacket M, Feigin V, Barber PA, Broad JB, Bonita R: Trends in ethnic disparities in stroke incidence in Auckland, New Zealand, during 1981 to 2003. Stroke 2006;37:56-62.

$\longrightarrow 9$ Bejot Y, Daubail B, Jacquin A, Durier J, Osseby GV, Rouaud O, Giroud M: Trends in the incidence of ischaemic stroke in young adults between 1985 and 2011: the Dijon Stroke Registry. J Neurol Neurosurg Psychiatry 2014;85: 509-513.

10 Adams HP Jr, Bendixen BH, Kappelle LJ, Biller J, Love BB, Gordon DL, Marsh EE: Classification of subtype of acute ischemic stroke. Definitions for use in a multicenter clinical trial. Toast. Trial of Org 10172 in Acute Stroke Treatment. Stroke 1993;24:3541.

11 Kolominsky-Rabas PL, Sarti C, Heuschmann PU, Graf C, Siemonsen S, Neundoerfer B, Katalinic A, Lang E, Gassmann KG, von Stockert TR: A prospective community-based study of stroke in Germany - the Erlangen Stroke Project (ESPro) incidence and case fatality at 1, 3, and 12 months. Stroke 1998;29: 2501-2506.

12 Kolominsky-Rabas PL, Weber M, Gefeller O, Neundoerfer B, Heuschmann PU: Epidemiol- ogy of ischemic stroke subtypes according to TOAST criteria: incidence, recurrence, and long-term survival in ischemic stroke subtypes: a population-based study. Stroke 2001; 32:2735-2740.

13 Hatano S: Experience from a multicentre stroke register: a preliminary report. Bull World Health Organ 1976;54:541-553.

14 Feigin V, Hoorn SV: How to study stroke incidence. Lancet 2004;363:1920.

15 Doll R, Cook P: Summarizing indices for comparison of cancer incidence data. Int J Cancer 1967;2:269-279.

16 Fay MP, Feuer EJ: Confidence intervals for directly standardized rates: a method based on the gamma distribution. Stat Med 1997;16: 791-801.

17 Feigin VL, Lawes CM, Bennett DA, BarkerCollo SL, Parag V: Worldwide stroke incidence and early case fatality reported in 56 population-based studies: a systematic review. Lancet Neurol 2009;8:355-369.

18 Kulesh SD, Filina NA, Frantava NM, Zhytko NL, Kastsinevich TM, Kliatskova LA, Shumskas MS, Hilz MJ, Schwab S, Kolominsky-Rabas PL: Incidence and case-fatality of stroke on the East border of the European union: the Grodno Stroke Study. Stroke 2010;41:27262730.

19 Kulesh SD, Kastsinevich TM, Kliatskova LA, Sauchanka ME, Filina NA, Shumskas MS, Schwab S, Hilz MJ, Kolominsky-Rabas PL: Long-term outcome after stroke in Belarus: the Grodno stroke study. Stroke 2011;42: 3274-3276.

20 Benatru I, Rouaud O, Durier J, Contegal F, Couvreur G, Bejot Y, Osseby GV, Ben Salem D, Ricolfi F, Moreau T, Giroud M: Stable stroke incidence rates but improved case-fatality in Dijon, France, from 1985 to 2004. Stroke 2006;37:1674-1679. 
21 Wolf-Maier K, Cooper RS, Banegas JR, Giampaoli S, Hense HW, Joffres M, Kastarinen M, Poulter N, Primatesta P, Rodriguez-Artalejo F, Stegmayr B, Thamm M, Tuomilehto J, Vanuzzo D, Vescio F: Hypertension prevalence and blood pressure levels in 6 European countries, Canada, and the United States. JAMA 2003;289:2363-2369.

-22 Diederichs C, Neuhauser H: Regional variations in hypertension prevalence and management in Germany: results from the German Health Interview and Examination Survey (DEGS1). J Hypertens 2014;32:14051413; discussion 1414.
23 Prugger C, Heidrich J, Wellmann J, Dittrich $\mathrm{R}$, Brand SM, Telgmann R, Breithardt G, Reinecke H, Scheld H, Kleine-Katthofer P, Heuschmann PU, Keil U: Trends in cardiovascular risk factors among patients with coronary heart disease: results from the EUROASPIRE I, II, and III surveys in the Munster region. Dtsch Arztebl Int 2012;109: 303-310.

24 de Looze M, ter Bogt T, Hublet A, Kuntsche E, Richter M, Zsiros E, Godeau E, Vollebergh W: Trends in educational differences in adolescent daily smoking across Europe, 2002-10. Eur J Public Health 2013;23:846852.
5 Ay H, Furie KL, Singhal A, Smith WS, Sorensen AG, Koroshetz WJ: An evidencebased causative classification system for acute ischemic stroke. Ann Neurol 2005;58:688697.

26 Hajat C, Heuschmann PU, Coshall C, Padayachee S, Chambers J, Rudd AG, Wolfe $\mathrm{CD}$ : Incidence of etiological subtypes of stroke in a multi-ethnic population based study: the South London Stroke Register. J Neurol Neurosurg Psychiatry 2010;82:527533. 